\title{
Search for high column density systems with gamma ray bursts (Research Note)
}

\author{
H. Hirashita ${ }^{1,2, \star}$, H. Shibai $^{2}$, and T. T. Takeuchi ${ }^{3, \star \star}$ \\ 1 SISSA/International School of Advanced Studies, via Beirut 4, 34014 Trieste, Italy \\ 2 Graduate School of Science, Nagoya University, Furo-cho, Chikusa-ku, Nagoya, 464-8602, Japan \\ e-mail: [hirashita;shibai]@u.phys.nagoya-u.ac.jp \\ 3 Laboratoire d'Astrophysique de Marseille, Traverse du Siphon BP 8, 13376 Marseille Cedex 12, France \\ e-mail: tsutomu.takeuchi@oamp.fr
}

Received 10 February 2005 / Accepted 28 February 2006

ABSTRACT

\begin{abstract}
We investigate the possibility of searching for metal-poor high column density $\left(\gtrsim 10^{23} \mathrm{~cm}^{-2}\right)$ clouds at high redshift $(z)$ by using gamma ray burst afterglows. Such clouds could be related to primeval galaxies that may cause a burst of star formation. We show that a large part of hydrogen is in molecular form in such a high column density environment. Therefore, hydrogen molecules $\left(\mathrm{H}_{2}\right)$ rather than hydrogen atoms should be searched for. Then we show that infrared $\mathrm{H}_{2}$ lines are detectable for metal-poor $(\leqslant 0.01$ solar metallicity) high column density ( $\left.\log N_{\mathrm{H}}\left[\mathrm{cm}^{-2}\right] \gtrsim 23.5\right)$ systems at high- $z$ without suffering dust extinction. The optical properties of dust in infrared could also be constrained by observations of high column density systems. Some possible scenarios for producing high column density systems are finally discussed in the context of galaxy evolution.
\end{abstract}

Key words. galaxies: evolution - galaxies: ISM - infrared: ISM - ISM: dust, extinction - ISM: lines and bands ISM: molecules

\section{Introduction}

Intergalactic and interstellar gas is a main component of baryonic matter, and it occupies most of the volume in the Universe. Gas with various column densities has been detected as line absorption of the background quasi-stellar objects (QSOs). The strong $\operatorname{Ly} \alpha$ absorption feature is often used to identify absorbing clouds, which are called Ly $\alpha$ clouds. In particular, if the H I column density is larger than $2 \times 10^{20} \mathrm{~cm}^{-2}$, the absorbing system is called damped Ly $\alpha$ clouds (DLAs) because the continuum of background QSOs is completely damped by the Ly $\alpha$ absorption (e.g., Wolfe et al. 1986).

So far, the hydrogen column density has been sampled up to $\log N_{\mathrm{H}} \lesssim 22$ (in this paper, we show all the column densities in units of $\mathrm{cm}^{-2}$ ). It is difficult to detect systems with higher column density, since dust severely extinguishes the background objects. Fall et al. (1989) show that QSOs behind DLAs tend to be redder than those without DLAs. This result strongly suggests that the dust contained in high column density systems extinguishes the background light (but see Murphy \& Liske 2004). If $\log N_{\mathrm{H}} \gtrsim 22$, the effects of extinction become significant and a large fraction of absorption systems can be missed (e.g., Vladilo \& Péroux 2005).

Schaye (2001) proposes another possibility for missing high column density systems. He shows that a large fraction of hydrogen is in molecular form if $\log N_{\mathrm{H}} \gtrsim 22$. Such molecular clouds

* Postdoctoral Fellow of the Japan Society for the Promotion of Science (JSPS). Present address: Center for Computational Sciences, University of Tsukuba, Tsukuba 305-8577, Japan.

$\star \star$ Postdoctoral Fellow of the JSPS for Research Abroad. Present address: Astronomical Institute, Tohoku University, Aramaki, Aoba-ku, Sendai 980-8578, Japan. may not be sampled by $\operatorname{Ly} \alpha$ lines of HI, so it is necessary to search for molecular hydrogen $\left(\mathrm{H}_{2}\right)$. Shibai et al. (2001, hereafter S01) investigate the observability of $\mathrm{H}_{2}$ in absorption for high column density systems. They focus on near-infrared (NIR) vib-rotational lines, because dust extinction is less severe in the NIR than in the optical and ultraviolet (UV). Considering typical QSOs as background sources, they show that the high column density systems with an $\mathrm{H}_{2}$ column density of $\log N_{\mathrm{H}_{2}} \gtrsim 23$ could be observed without being extinguished by dust if the dustto-gas ratio is less than 1 percent of the Galactic value.

However, QSOs are usually sampled in the optical wavelength, where dust extinction is severe. Ellison et al. (2001) investigated a sample of radio-selected QSOs and conclude that dust-induced bias in optical samples is small. Therefore, the significance of extinction bias is still being debated. In this paper, we consider the possibility of using gamma-ray burst (GRB) afterglows as background sources. Since GRB samples are defined in a wavelength not affected by dust extinction, we expect less extinction bias in GRB samples than in QSO samples. Indeed some DLAs are found to lie in the sightline of GRBs (e.g., Vreeswijk et al. 2004) and some GRBs are surrounded by high column density gas (e.g., Piro et al. 2005). Therefore, it may be possible to use NIR continua of GRB afterglows to detect NIR $\mathrm{H}_{2}$ lines.

Recently, Inoue et al. (2006) investigated the possibility of detecting the absorption lines originating from protostellar cores by using background GRB afterglows. They find that radio observations of some metal absorption lines of GRB afterglows are useful for detecting high- $z$ protostellar gas. However, it is still interesting to detect directly $\mathrm{H}_{2}$ because $\mathrm{H}_{2}$ is considered as playing a central role in star formation in metal-poor environments 
(e.g., Mizusawa et al. 2004). Therefore, in spite of its difficulty, the possibility of detecting IR $\mathrm{H}_{2}$ lines is worth investigating.

Since $\mathrm{H}_{2}$ vib-rotational lines are generally weak, large infrared (IR) telescopes are necessary. The strongest vib-rotational $\mathrm{H}_{2}$ lines are normally those around the wavelength of $\lambda \sim 2 \mu \mathrm{m}$. Those lines shift to the mid-IR (MIR) regime if we observe high- $z$ clouds. In MIR, it is essential to avoid the emissions of both the atmosphere and telescope. Thus, a cooled space telescope is ideal. There are indeed suitable future missions planned currently, such as the Space Infrared Telescope for Cosmology and Astrophysics (SPICA) ${ }^{1}$. Herschel ${ }^{2}$ is also a planned space mission in IR (but not cooled), and the same kind of targets can be accessible if the background sources are luminous enough. In NIR $(\lambda \lesssim 5 \mu \mathrm{m})$, the James Webb Space Telescope $(J W S T)^{3}$ is more sensitive than the SPICA, and is appropriate for $\mathrm{H}_{2}$ absorption at $z \lesssim 1.5$.

First, in Sect. 2 we explain the method of calculating $\mathrm{H}_{2}$ absorption line luminosity. Our results are shown in Sect. 3, and some relevant physical and observational issues are discussed in Sect. 4. Our conclusions are given in Sect. 5.

\section{2. $\mathrm{H}_{2}$ absorption lines}

\subsection{Absorption line flux}

We follow $\mathrm{S} 01$ for the formulation concerning IR $\mathrm{H}_{2}$ lines. We assume a uniform, cool gas cloud satisfying $k_{\mathrm{B}} T_{\mathrm{ex}} \ll h v$, where $k_{\mathrm{B}}$ is the Boltzmann constant, $T_{\mathrm{ex}}$ the excitation temperature, $h$ the Planck constant, and $v$ the frequency of an $\mathrm{H}_{2}$ line.

The optical depth of the line absorption, $\tau_{\text {line }}$, is estimated as

$\tau_{\text {line }} \simeq \frac{\lambda^{3}}{8 \pi}\left(\frac{g_{u}}{g_{\ell}}\right) A_{u \ell} N_{\ell} \frac{1}{\Delta v}$,

where subscripts $u$ and $\ell$ indicate the upper and lower levels of a transition, $g_{u}$ and $g_{\ell}$ are the degeneracy of each state, respectively, $A_{u \ell}$ is Einstein's $A$ coefficient, $N_{\ell}$ the column density of the molecules in the lower state, and $\Delta v$ the line width in units of velocity. If the instrumental wavelength resolution is larger than the physical line width, we should take $\Delta v=c / R$, where $R$ is the resolution defined by $R \equiv \lambda / \Delta_{\mathrm{i}} \lambda$ ( $\Delta_{\mathrm{i}} \lambda$ is the wavelength resolved by the instrument).

Next, we consider extinction by dust grains, whose optical depth at the wavelength $\lambda$ is denoted as $\tau_{\text {dust }}(\lambda)$. This is estimated as (Hirashita et al. 2005)

$\tau_{\text {dust }}(\lambda)=\mu m_{\mathrm{H}} N_{\mathrm{H}} \mathcal{D}\left\langle\sigma_{\mathrm{d}}(\lambda) / m_{\mathrm{d}}\right\rangle$,

where $\mu$ is the mean atomic mass per $\mathrm{H}$ atom (assumed to be 1.4), $m_{\mathrm{H}}$ the mass of a hydrogen atom, $N_{\mathrm{H}}$ the column density of hydrogen nuclei, $\mathcal{D}$ the dust-to-gas mass ratio, and $\left\langle\sigma_{\mathrm{d}}(\lambda) / m_{\mathrm{d}}\right\rangle$ the cross section of dust per unit dust mass as a function of wavelength. $I_{\text {line }}^{\text {abs }}$, as

Finally, we obtain the absorption line flux with extinction,

$I_{\text {line }}^{\text {abs }}=S_{v} \Delta v\left[1-\exp \left(-\tau_{\text {line }}\right)\right] \exp \left(-\tau_{\text {dust }}\right)$,

where $\Delta v(=v \Delta v / c)$ is the line width in units of frequency and $S_{v}$ the continuum flux (per frequency) of the IR source behind the cloud.

\footnotetext{
${ }^{1}$ http://wWw.ir.isas.ac.jp/SPICA/index.html

${ }^{2}$ http://astro.estec.esa.nl/SA-general/Projects/ Herschel/

${ }^{3}$ http://ngst.gsfc.nasa.gov/
}

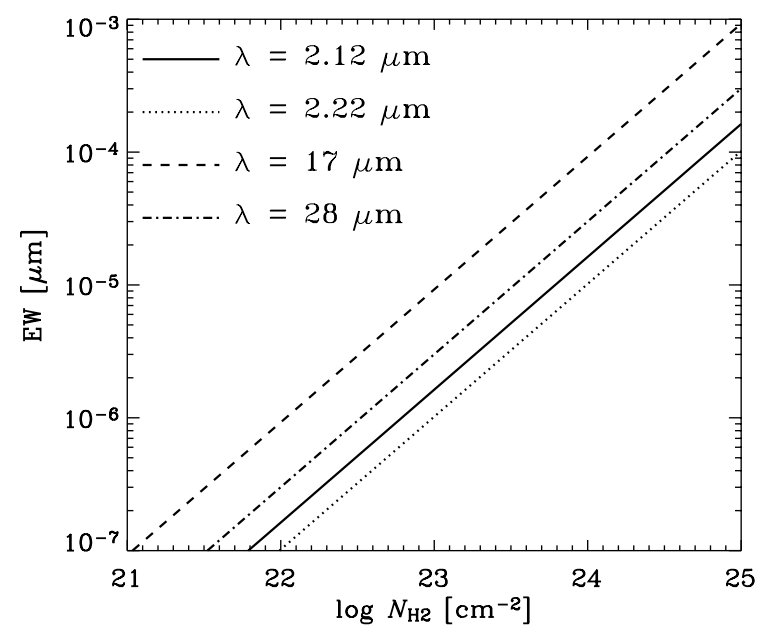

Fig. 1. Equivalent width $(E W)$ for various $\mathrm{H}_{2}$ lines as a function of $\mathrm{H}_{2}$ column density. The solid, dotted, dashed, and dot-dashed lines represent the line wavelengths of $\lambda=2.12,2.22,17$, and $28 \mu \mathrm{m}$, respectively.

We assume that all the molecular hydrogens are in the levels $J=0,1$ of the vibrational ground state $(v=0)$, since we are interested in the clouds whose excitation temperature is much lower than the excitation energy. The lines are summarized in $\mathrm{S} 01$. The ortho vs. para ratio is assumed to be $3: 1$; i.e., $N_{1}=(3 / 4) N_{\mathrm{H}_{2}}$ and $N_{0}=(1 / 4) N_{\mathrm{H}_{2}}$, where $N_{J}$ is the column density of $\mathrm{H}_{2}$ in the level $J$ and $N_{\mathrm{H}_{2}}$ the total hydrogen column density.

Observationally, it could be convenient to present the equivalent width, since it is not sensitive to the line width (or the spectral resolution). The equivalent width $W_{\lambda}$ given in wavelength units is expressed as

$W_{\lambda}=\frac{\lambda^{2}}{c}\left[1-\exp \left(-\tau_{\text {line }}\right)\right] \Delta v$.

We calculate $W_{\lambda}$ as a function of $N_{\mathrm{H}_{2}}$ as shown in Fig. 1. We should note that not only the equivalent width but also the suppression of continuum level by dust extinction is important for the absorption flux (Eq. (3)).

\subsection{Extinction models}

The optical depth of dust, $\tau_{\text {dust }}$, is proportional to the dust-to-gas ratio and to the hydrogen column density, if we fix $\left\langle\sigma_{\mathrm{d}}(\lambda) / m_{\mathrm{d}}\right\rangle$ as a function of $\lambda$ (Eq. (2)), which is taken from Hirashita et al. (2005). We adopt the Galactic extinction curve, which is consistent with Mathis (1990), unless otherwise stated. A different extinction curve is examined in Sect. 4.2. We assume that the Galactic dust-to-gas ratio, $\mathcal{D}_{\odot}$, is $6 \times 10^{-3}$, since we should adopt the values in Hirashita et al. (2005) for consistency. We define the normalized dust-to-gas ratio, $\kappa$, as

$\kappa \equiv \mathcal{D} / \mathcal{D}_{\odot}$.

\subsection{Background sources}

We focus on GRB afterglows as one of the most luminous sources in the Universe. If some dense clouds lie in the line of sight of GRBs, we can have a chance of detecting these foreground clouds in absorption. For the flux of GRB afterglows, we adopt a commonly used model with synchrotron radiation from a relativistic shock (Sari et al. 1998; Ciardi \& Loeb 2000). Here 
we apply a set of values adopted by Inoue et al. (2004): a magnetic energy fraction of $\epsilon_{B}=0.1$, an electron energy fraction of $\epsilon_{\mathrm{e}}=0.2$, a spherical shock energy of $E=10^{52} \mathrm{erg}$, an ambient gas number density of $n=10 \mathrm{~cm}^{-3}$, and a power-law index of the electron energy distribution of $p=2.5$. For the timescale, we put $t=1 / 24$ day $(1 \mathrm{~h})$, and the observational detection limits are also calculated with 1 -h integration.

We do not include the redshift dependence of GRB luminosity. GRBs may be brighter at high $z$ than the local Universe (e.g., Lloyd-Ronning et al. 2002; Yonetoku et al. 2004). In this case, detection of high- $z$ absorption lines becomes easier.

\section{Results}

We consider a velocity width of $\Delta v=100 \mathrm{~km} \mathrm{~s}^{-1}$, motivated by the future cooled space telescopes, such as SPICA with $R \sim$ 3000. Accordingly, the frequency resolution becomes $\Delta v=v / R$. Then, $S_{v} \Delta v$ is estimated as

$S_{v} \Delta v=1.0 \times 10^{-18}\left(\frac{S}{1 \mathrm{mJy}}\right)\left(\frac{\lambda}{1 \mu \mathrm{m}}\right)^{-1}\left(\frac{R}{3000}\right)^{-1} \mathrm{~W} \mathrm{~m}^{-2}$.

We assume that the all the hydrogen atoms are in the molecular form (i.e., $N_{\mathrm{H}}=2 N_{\mathrm{H}_{2}}$ ), an assumption discussed in Sect. 4.1.

We note that $\mathrm{S} 01$ scale the extinction by $Z$ (metallicity normalized to the solar value). We have confirmed that if we adopt $\kappa=Z$, the difference in $\tau_{\text {dust }}$ is less than a factor of 2 . As shown by $\mathrm{S} 01$, the $2.12 \mu \mathrm{m}$ absorption line is the strongest, and the dust obscuration does not affect the line detection if $\kappa \lesssim 0.01$. Therefore, we concentrate on the $2.12 \mu \mathrm{m}$ line with $\kappa=0.01$ in this section.

As stated by S01, it is quite difficult to detect absorption lines where optical depth is less than 0.01 , since an extremely high signal-to-noise ratio would be required to detect an absorption with $\tau_{\text {line }}<0.01$. The $\mathrm{H}_{2}$ column density that satisfies $\tau_{\text {line }}=0.01$ is listed in S01 for various lines. For the $2.12 \mu \mathrm{m}$ line, $N_{\mathrm{H}_{2}}$ should be higher than $4 \times 10^{23} \mathrm{~cm}^{-2}$ to satisfy $\tau_{\text {line }}>0.01$. Therefore, if we use the NIR $\mathrm{H}_{2}$ lines, we should target the absorption systems with $\log N_{\mathrm{H}_{2}} \gtrsim 23.5$. However, we should note that this condition is dependent on the line width. The sound speed of $\mathrm{H}_{2}$-rich cold clouds is generally much less than $100 \mathrm{~km} \mathrm{~s}^{-1}$. Therefore, if we had a facility that is sensitive enough with a high enough resolution, we could observe an absorption system whose $\mathrm{H}_{2}$ column density is lower than $10^{23.5} \mathrm{~cm}^{-2}$.

In Fig. 2, we show the $2.12 \mu \mathrm{m}$ absorption fluxes as a function of $N_{\mathrm{H}_{2}}$ for various background fluxes. The typical detection limit expected for future IR cooled telescopes such as SPICA is also shown $\left(5 \times 10^{-21} \mathrm{~W} \mathrm{~m}^{-2}\right.$ for $5 \sigma$ detection with an integration of $\sim 1 \mathrm{~h}$; Ueno et al. 2000; S01) as a representative observability of future facilities. With future space missions, high column density systems with $\log N_{\mathrm{H}_{2}} \sim 23.5-25$ can be investigated if the background source luminosity is $\gtrsim 10 \mathrm{mJy}$.

In order to show the flux level of GRB afterglows, we present Fig. 3, which shows the flux $\left(S_{v}\right)$ at the restframe wavelengths of $\lambda=2.1 \mu \mathrm{m}$ and $17 \mu \mathrm{m}$ (i.e., the observational wavelengths of $2.1\left(1+z_{\mathrm{GRB}}\right) \mu \mathrm{m}$ and $\left.17\left(1+z_{\mathrm{GRB}}\right) \mu \mathrm{m}\right)$, respectively, where $z_{\mathrm{GRB}}$ is the redshift of the GRB afterglow. We observe that the afterglows are more luminous at $2.1\left(1+z_{\mathrm{GRB}}\right) \mu \mathrm{m}$ than at $17\left(1+z_{\mathrm{GRB}}\right) \mu \mathrm{m}$. In reality, we should observe at $2.1(1+$ $\left.z_{\mathrm{abs}}\right) \mu \mathrm{m}$ or $17\left(1+z_{\mathrm{abs}}\right) \mu \mathrm{m}$, where $z_{\mathrm{abs}}$ is the redshift of a target absorption line system, but if $z_{\text {abs }}$ has the same order of magnitude as $z_{\mathrm{GRB}}$, the absorption flux does not change very much (the dependence on other parameters assumed in Sect. 2.3 is

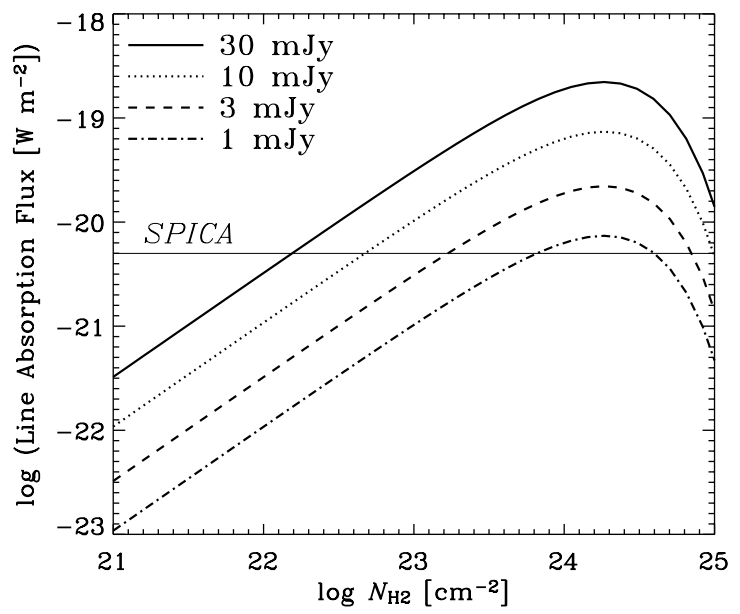

Fig. 2. The $2.12 \mu \mathrm{m}$ line absorption flux as a function of the column density of hydrogen molecules, and various continuum fluxes of the background sources. The solid, dotted, dashed, and dot-dashed curves correspond to the continuum fluxes of $30,10,3$, and $1 \mathrm{mJy}$, respectively. The horizontal thin solid line shows the expected detection limit of future cooled space telescopes such as SPICA ( $5 \sigma$ for an integration of $\sim 1 \mathrm{~h}$ ).

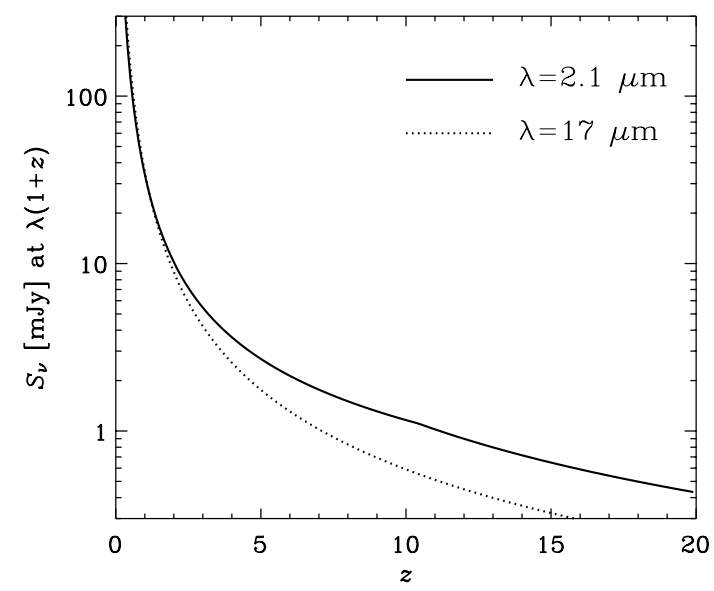

Fig. 3. Observed flux of a gamma-ray burst afterglow as a function of redshift. The solid and dotted lines correspond to the restframe wavelengths of 2.12 and $17 \mu \mathrm{m}$, i.e., observational wavelengths of $2.12(1+z)$ and $17(1+z) \mu \mathrm{m}$, respectively.

larger). Considering that the $2.1 \mu \mathrm{m}$ absorption line is stronger than the other lines, observations at $2.1\left(1+z_{\text {abs }}\right) \mu \mathrm{m}$ are effective of tracing high column density $\left(\log N_{\mathrm{H}_{2}} \sim 23.5-25\right)$ systems (see S01 for the other lines).

The dependence on the dust-to-gas ratio is also shown for the background flux of $10 \mathrm{mJy}$ (Fig. 4). The absorption line fluxes with $\kappa=0.0001,0.001,0.01$, and 1 , are examined. We see that if $\kappa>0.1$, we can detect the $\mathrm{NIR}_{2}$ line if $\log N_{\mathrm{H}_{2}} \gtrsim 23$.

\section{Discussions and observational strategies}

\subsection{High column density systems in cosmological scenarios}

\subsubsection{Protogalactic disks}

Collapsed gas in a dark matter halo may form a disk because of the initial angular momentum. If we observe a GRB in a nearly edge-on disk, we could identify the disk as a high column density system. If a galactic disk has a typical circular velocity of $100 \mathrm{~km} \mathrm{~s}^{-1}$, the typical mass $\left(M_{\text {disk }}\right)$ and radius $\left(r_{\text {disk }}\right)$ of the 


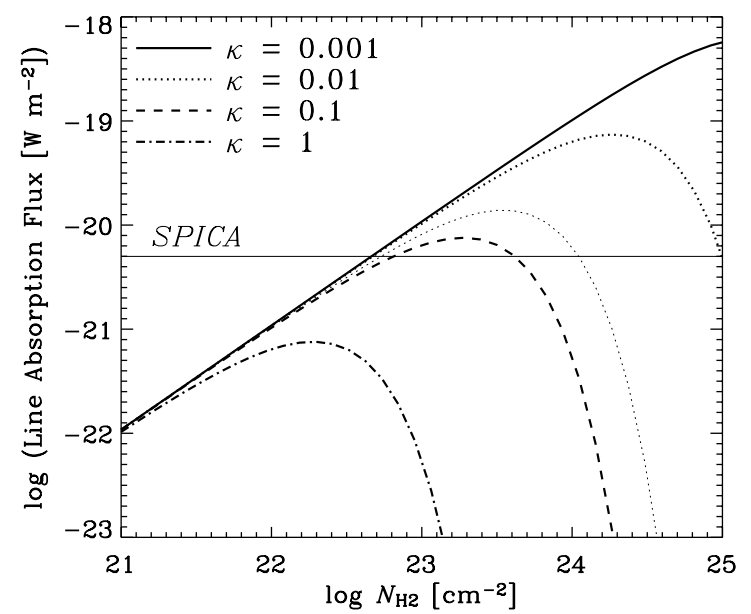

Fig. 4. Same as Fig. 2, but for various dust-to-gas ratios ( $\kappa$ is the dust-togas ratio normalized to the Galactic value). The continuum fluxes of the background sources is assumed to be $10 \mathrm{mJy}$. The solid, dotted, dashed, and dot-dashed lines correspond to $\kappa=0.01,0.1$, 1 , and 0.001 , respectively. The lower dotted line represents the result with the H05b extinction curve, while the other lines adopt the Galactic extinction curve.

disk should be $M_{\text {disk }} \sim 2 \times 10^{9} M_{\odot}$ and $r_{\text {disk }} \sim 6 \mathrm{kpc}$ if it forms at $z \sim 3$ (Hirashita et al. 2003). With this mass and size, the typical hydrogen column density along the galactic disk is $\sim n_{\mathrm{H}} r_{\text {disk }}$. Using the relation $M_{\text {disk }} \sim \pi r_{\text {disk }}^{2} H \mu m_{\mathrm{H}} n_{\mathrm{H}}$, where $H$ is the disk thickness, we obtain

$$
\begin{aligned}
n_{\mathrm{H}} r_{\text {disk }} \sim & 1 \times 10^{23}\left(\frac{M_{\text {disk }}}{2 \times 10^{9} M_{\odot}}\right)\left(\frac{r_{\text {disk }}}{6 \mathrm{kpc}}\right)^{-1} \\
& \times\left(\frac{H}{100 \mathrm{pc}}\right)^{-1} \mathrm{~cm}^{-2} .
\end{aligned}
$$

Therefore, a typical galactic disk could be observed as a high column density system if a GRB lies within or behind the disk and if a significant fraction of the gas is in molecular form.

As stated in Sect. 3, the dust-to-gas ratio must be $\lesssim 10^{-2}$ times the Galactic value (i.e., $\mathcal{D} \lesssim 6 \times 10^{-5}$ ). With the typical disk mass mentioned above $\left(2 \times 10^{9} M_{\odot}\right)$, the corresponding dust mass is $M_{\text {dust }} \lesssim 10^{5} M_{\odot}$. We estimate the timescale of the dust enrichment up to this level. Galactic disks observed as DLAs can be considered as objects with a star formation rate of $\sim 0.1-1 M_{\odot} \mathrm{yr}^{-1}$ (Hirashita \& Ferrara 2005, hereafter HF05). Using the Salpeter initial mass function, we obtain the supernova rate of $\sim 10^{-3}-10^{-2} \mathrm{yr}^{-1}$. Assuming that around $0.5 M_{\odot}$ of the dust is formed in a SN (Todini \& Ferrara 2001; Nozawa et al. 2003), we obtain a typical dust formation rate of $\sim 5 \times 10^{-4}-5 \times$ $10^{-3} M_{\odot} \mathrm{yr}^{-1}$. Thus, in order to accumulate the dust mass estimated above, a timescale of $2 \times 10^{7}-2 \times 10^{8} \mathrm{yr}$ is required, which is much larger than the lifetime of the GRB progenitors (Heger et al. 2003).

It is observationally known that some DLAs have dust-to-gas ratio of $\kappa \sim 10^{-2}$ (Pettini et al. 1997; Ledoux et al. 2003; HF05). This observational evidence strongly suggests that galactic disks with $\kappa \sim 10^{-2}$ exist at high redshift.

\subsubsection{Supergiant molecular complexes}

In some starburst galaxies such as the Antennae (Wilson et al. 2003) and Arp 229 (Casoli et al. 1999), supergiant molecular complexes are observed. In those galaxies, molecular gas of $\sim 10^{9} M_{\odot}$ is concentrated in a $1-\mathrm{kpc}^{2}$ area. This indicates an

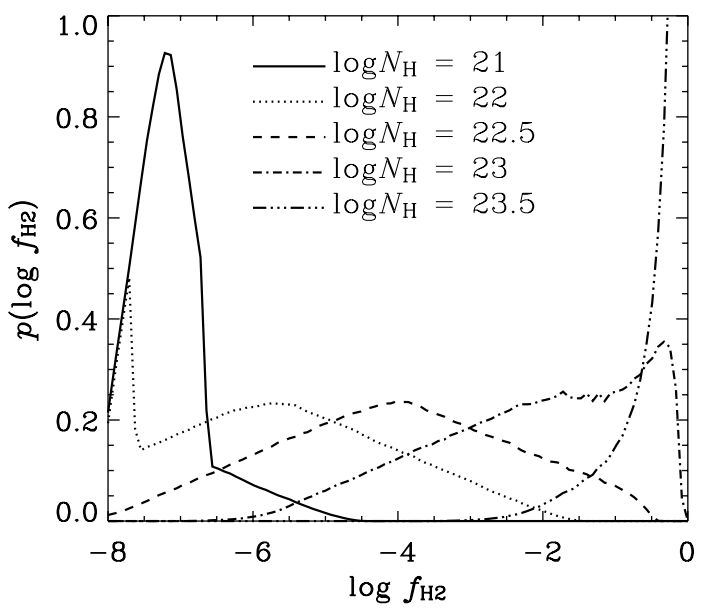

Fig. 5. Probability distribution function of the molecular fraction $\left(\log f_{\mathrm{H}_{2}}\right)$ for various hydrogen column densities. The solid, dotted, dashed, dot-dashed, and dot-dot-dot-dashed lines correspond to $\log N_{\mathrm{H}}\left(\mathrm{cm}^{-2}\right)=21,22,22.5,23$, and 23.5 , respectively. The dust-togas ratio normalized to the Galactic value is assumed to be $\kappa=0.01$.

$\mathrm{H}_{2}$ column density that is as high as $\sim 10^{23} \mathrm{~cm}^{-2}$ (and a hydrogen number density of $n_{\mathrm{H}} \sim 30 \mathrm{~cm}^{-3}$ ). Arp 220 seems to have a comparable amount of gas in more concentrated region (Sakamoto et al. 1999). Thus, the $\mathrm{H}_{2}$ column density in a starbursting region can exceed several times $10^{23} \mathrm{~cm}^{-2}$. This column density meets the requirement for detecting $\mathrm{H}_{2}$ in absorption.

HF05 have recently developed a method for calculating a probable range of $f_{\mathrm{H}_{2}}$ under a given range of physical quantities concerning ISM. Their assumption is that $f_{\mathrm{H}_{2}}$ is determined by the equilibrium between formation on dust grains and destruction by dissociating photons. The probability density function of $\log f_{\mathrm{H}_{2}}, p\left(\log f_{\mathrm{H}_{2}}\right)$, is calculated by using the method described in Appendix B of HF05. As a first guess, we start from the ranges of quantities derived for $\mathrm{H}_{2}$-detected DLAs by HF05: $1.5<\log n\left[\mathrm{~cm}^{-3}\right]<2.5,1.5<\log T[\mathrm{~K}]<3$, and $0.5<\log \chi<1.5$, where $n$ is the number density of gas particles, $T$ the gas temperature, and $\chi$ the UV interstellar radiation field intensity normalized to the Galactic value taken from Habing (1968). We adopt $\kappa=0.01$ for the dust-to-gas ratio (Sect. 3). In Fig. 5, we show $p\left(\log f_{\mathrm{H}_{2}}\right)$ for various $N_{\mathrm{H}}$. From Fig. 5, we observe that the gas becomes almost fully molecular for $\log N_{\mathrm{H}} \gtrsim 23$ even if the dust-to-gas ratio is only 1 percent of the Galactic value $(\kappa \sim 0.01)$. This is consistent with the conclusion by Schaye (2001) that a large part of hydrogen is in molecular form at $\log N_{\mathrm{H}} \gtrsim 22$. We stress that at $\kappa \sim 0.01 \mathrm{H}_{2}$ formation is accelerated and possibly leads to a burst of star formation (Hirashita \& Ferrara 2002). Therefore, our proposed observation is important because we will trace the reservoir of gas clouds that might cause a starburst. Indeed Iliev et al. (2006) investigate the fate of those clouds proposed by HF05 and conclude that they can finally collapse even in the presence of UV radiation.

\subsection{Extinction curves}

Some observations show that the extinction curves of high- $z$ galaxies are different from local ones (Maiolino et al. 2004). Therefore, it is interesting to examine the extinction curve derived for high- $z$ galaxies. We adopt the theoretical curve of Hirashita et al. (2005), and adopt model b (based on the dust production in an unmixed SNe II; Nozawa et al. 2003) of Hirashita et al. (2005), which nearly reproduces the observational high- $z$ 
extinction curve of Maiolino et al. (2004). This model is labeled as H05b (see Takeuchi et al. 2005 for the NIR part of the extinction curve).

We show the absorption flux of the $2.12 \mu \mathrm{m}$ line for the H05b extinction curve in Fig. 4, assuming that the dust-to-gas ratio is 0.01 times the Galactic value. Comparing the upper and lower dotted lines (corresponding to the Galactic and $\mathrm{H} 05 \mathrm{~b}$ extinction curves, respectively), we observe that the difference in the extinction curve does not affect the absorption line flux for $\log N_{\mathrm{H}_{2}} \lesssim 23$, since the optical depth of dust is small. However, the difference in the extinction becomes significant for $\log N_{\mathrm{H}_{2}} \gtrsim 23.5$. In H05b the system becomes more opaque for $\log N_{\mathrm{H}_{2}} \gtrsim 24$ than in the Galactic extinction case. This sensitive dependence on the extinction could also be used to constrain the IR extinction curves at high $z$ (see also Vergani et al. 2004).

\subsection{Number estimate}

There is no observational constraint on the number of high column density systems with $\log N_{\mathrm{H}} \gtrsim 22$. One simple way to estimate the number is to extrapolate the distribution of $N_{\mathrm{H}}$ sampled up to $\log N_{\mathrm{H}} \sim 22$, although Vladilo \& Péroux (2005) argue that the distribution should not be extrapolated in the range of column densities not sampled by the observation. After correcting the distribution for dust extinction, Vladilo \& Péroux (2005) indicate that $\log f\left(N_{\mathrm{H}} \sim 10^{22} \mathrm{~cm}^{-2}\right) \sim-23.5$, where $f\left(N_{\mathrm{H}}\right) \mathrm{d} N_{\mathrm{H}} \mathrm{d} X$ represents the number of absorbers along a random line of sight with hydrogen column densities between $N_{\mathrm{H}}$ and $N_{\mathrm{H}}+\mathrm{d} N_{\mathrm{H}}$ and redshift paths between $X$ and $X+\mathrm{d} X$ (e.g., Péroux et al. 2003). By assuming $f\left(N_{\mathrm{H}}\right) \propto N_{\mathrm{H}}^{-\beta}$ with $\beta=1.5$ (Vladilo \& Péroux 2005), we obtain $\log f\left(N_{\mathrm{H}}\right) \sim-25.8$ at $\log N_{\mathrm{H}} \sim 23.5$. At $z=2, X \simeq 4.4$, and if we take the interval of $N_{\mathrm{H}}$ as $\sim 10^{23} \mathrm{~cm}^{-2}$, we obtain the number of absorbers in a random line of sight as $\sim 10^{-2.8}$. Therefore, around 600 lines of sight are needed to detect a high column density object. If a large part of those sources are favorable for $\mathrm{H}_{2}$ formation as suggested in Sect. 4.1, we can detect $\mathrm{H}_{2}$ lines every $~ 600$ GRBs.

For example, the sample of Schmidt (2001) consists of 1391 GRBs in a period of 5.9 yr. Their analysis indicates that about 500 GRBs are around $z \sim 2$. Therefore, it is possible that about 100 GRBs are identified around $z \sim 2$ per year. Therefore, we could find 0.2 GRBs associated with high column density systems per year. If we consider an improvement of the sensitivity in the future, we expect a larger number.

\section{Conclusions}

In this paper, we have investigated the possibility of detecting IR $\mathrm{H}_{2}$ absorption lines from dust-poor high column density $\left(\log N_{\mathrm{H}} \gtrsim 23.5\right)$ clouds at high- $z$. The $\mathrm{H}_{2}$ molecule is a unique tracer of high column density clouds, because hydrogen is considered to be in a molecular form even at extremely low dust-to-gas ratios $(\kappa \sim 0.01$, where $\kappa$ is the dust-to-gas ratio normalized to the Galactic value) (Sect. 4.1). We have shown that if $\kappa<0.1$, we can observe $\mathrm{H}_{2}$ absorption lines of gas whose column density is $\log N_{\mathrm{H}_{2}} \gtrsim 23.5$. In particular, if $\kappa \lesssim 0.01$, clouds with $\log N_{\mathrm{H}_{2}} \sim 23.5-25$ are accessible (Sect. 3; Fig. 4). Since missions in the near future such as SPICA will access GRB afterglows up to $z \sim 2$ in the IR regime, the search for metal-poor (or dust-poor) galaxies at $z \lessgtr 2$ is an important scientific target for IR telescopes in the near future (Sect. 4.3). We should note that some bright GRBs at high- $z$ can be utilized to trace higher- $z$ primeval galaxies.

Acknowledgements. We are grateful to A. Ferrara, C. Péroux, P. Petitjean, and T. Nakamura for stimulating discussions. We acknowledge several helpful comments of the anonymous referee. H.H. and T.T.T. have been supported by the Research Fellowship of the Japan Society for the Promotion of Science for Young Scientists.

\section{References}

Casoli, F., Willaime, M.-C., Viallefond, F., \& Gerin, M. 1999, A\&A, 346, 663 Ciardi, B., \& Loeb, A. 2000, ApJ, 540, 687

Ellison, S. L., Yan, K., Hook, I. M., et al. 2001, A\&A, 379, 393

Fall, S. M., Pei, Y. C., \& McMahon, R. G. 1989, ApJ, 341, L5

Habing, H. J. 1968, Bull. Astron. Inst. Netherlands, 19, 421

Heger, A., Fryer, C. L., Langer, N., \& Hartmann, D. H. 2003, ApJ, 591, 288

Hirashita, H., \& Ferrara, A. 2002, MNRAS, 337, 921

Hirashita, H., \& Ferrara, A. 2005, MNRAS, 356, 1529 (HF05)

Hirashita, H., Ferrara, A., Wada, K., \& Richter, P. 2003, MNRAS, 341, L18

Hirashita, H., Nozawa, T., Kozasa, T., Ishii, T. T., \& Takeuchi, T. T. 2005, MNRAS, 357, 1077

Iliev, I. T., Hirashita, H., \& Ferrara, A. 2006, MNRAS, in press Inoue, A. K., Yamazaki, R., \& Nakamura, T. 2004, ApJ, 601, 644 Inoue, S., Omukai, K., \& Ciardi, B. 2006, MNRAS, submitted Ledoux, C., Petitjean, P., \& Srianand, R. 2003, MNRAS, 346, 209 Lloyd-Ronning, N. M., Fryer, C. L., \& Ramirez-Ruiz, E. 2002, ApJ, 574, 554 Maiolino, R., Schneider, R., Oliva, E., et al. 2004, Nature, 431, 533 Mathis, J. S. 1990, ARA\&A, 28, 37

Mizusawa, H., Nishi, R., \& Omukai, K. 2004, PASJ, 56, 487

Murphy, M. T., \& Liske, J. 2004, MNRAS, 354, L31

Nozawa, T., Kozasa, T., Umeda, H., et al. 2003, ApJ, 598, 785

Péroux, C., McMahon, R. G., Storrie-Lombardi, L. J., \& Irwin, M. J. 2003, MNRAS 346, 1103

Pettini, M., King, D. L., Smith, L. J., \& Hunstead, R. W. 1997, ApJ, 478, 536

Piro, L., De Pasquale, M., Soffitta, P., et al. 2005, ApJ, 623, 314

Sakamoto, K., Scoville, N. Z., Yun, M. S., et al. 1999, ApJ, 514, 68

Sari, R., Piran, T., \& Narayan, R. 1998, ApJ, 497, L17

Schaye, J. 2001, ApJ, 562, L95

Schmidt, M. 2001, ApJ, 552, 36

Shibai, H., Takeuchi, T. T., Rengarajan, T. N., \& Hirashita, H. 2001, PASJ, 53, 589 (S01)

Takeuchi, T. T., Ishii, T. T., Nozawa, T., Kozasa, T., \& Hirashita, H. 2005, MNRAS, 362, 592

Todini, P., \& Ferrara, A. 2001, MNRAS, 325, 726

Ueno, M., Matsuhara, H., Murakami, H., \& Nakagawa, T. 2000, in Mid- and FarInfrared Astronomy and Space Missions, ed. T. Matsumoto, \& H. Shibai, ISAS Report, SP-14, 189

Vergani, S. D., Molinari, E., Zerbi, F. M., \& Chincarini, G. 2004, A\&A, 415, 171

Vreeswijk, P. M., Ellison, S. L., Ledoux, C., et al. 2004, A\&A, 419, 927

Vladilo, G., \& Péroux, C. 2005, A\&A, 444, 461

Wilson, C. D., Scoville, N., Madden, S., \& Charmandaris, V. 2003, ApJ, 599, 1049

Wolfe, A. M., Turnshek, D. A., Smith, H. E., \& Cohen, R. D. 1986, ApJS, 61, 249

Yonetoku, D., Murakami, T., Nakamura, T., et al. 2004, ApJ, 609, 935 